\title{
FLAT FUNCTORS AND FREE EXACT CATEGORIES
}

\author{
HONGDE HU \\ (Received 7 October 1993; revised 7 February 1994) \\ Communicated by R. H. Street \\ Dedicated to Peter Freyd on his 60th birthday
}

\begin{abstract}
Let $\mathbf{C}$ be a small category with weak finite limits, and let Flat $(\mathbf{C})$ be the category of flat functors from $\mathbf{C}$ to the category of small sets. We prove that the free exact completion of $\mathbf{C}$ is the category of set-valued functors of Flat $(\mathbf{C})$ which preserve small products and filtered colimits. In case $\mathbf{C}$ has finite limits, this gives A. Carboni and R. C. Magno's result on the free exact completion of a small category with finite limits.
\end{abstract}

1991 Mathematics subject classification (Amer. Math. Soc.): 03G30, 18B25, 18E10, 18G05.

\section{Introduction}

Let $\mathbf{C}$ be a small lex (that is, finitely complete) category. It is well-known that there is a free-exact completion of $\mathbf{C}$ (see $[13,4,5,11,8]$ ). That is, there are an exact category $\mathbf{D}$ and a lex functor $F: \mathbf{C} \rightarrow \mathbf{D}$ such that $F$ has the following universal property: for any exact category $\mathbf{B}$, the functor $F^{*}: \operatorname{Reg}(\mathbf{D}, \mathbf{B}) \rightarrow \operatorname{Lex}(\mathbf{C}, \mathbf{B})$ given by the composition with $F$ is an equivalence of categories; here $\operatorname{Reg}(\mathbf{D}, \mathbf{B})$ is the category of regular functors from $\mathbf{D}$ to $\mathbf{B}$. The fundamental construction of A. Carboni and R. C. Magno gives an explicit description of this completion by adding as new objects the equivalence classes of pseudo-equivalence relations in $\mathbf{C}$ and as new arrows the suitable classes of compatible maps (see [4]). Recently, M. Makkai has shown that the free exact completion of $C$ is equivalent to $\prod$ Filt(Lex $(C$, Set $)$, Set), the category of functors from Lex $(\mathbf{C}$, Set) to Set that preserve products and filtered colimits (see $[8,11])$; here Set is the category of small sets.

This work was partially supported by a special research grant of the Faculty of Arts at York University.

(C) 1996 Australian Mathematical Society $0263-6115 / 95 \$ A 2.00+0.00$ 
The purpose of this paper is to generalize the above-mentioned result to a small category $\mathbf{C}$ with weak finite limits. The significance of the existence of weak finite limits for embedding theorems is already indicated in Freyd's early work [7].

For a small category $\mathbf{C}$, let $\mathbf{C}^{*}=$ Flat $(\mathbf{C})$ be the category of all flat functors from $\mathbf{C}$ to Set. The categories of the form $\mathbf{C}^{*}$ are known to be finitely accessible (see $\left.[9,12]\right)$. In case $\mathbf{C}$ has weak finite limits, they are exactly the finitely accessible categories with small products (see Theorem 1.7). Furthermore, for a category $\mathbf{A}$ with small products and filtered colimits, let $\mathbf{A}^{+}=\prod$ Filt(A, Set) be the category of functors from $\mathbf{A}$ to Set that preserve small products and filtered colimits. The fact that, in Set, small products and filtered colimits commute with finite limits and regular epimorphisms gives that $\mathbf{A}^{+}$is Barr-exact. For $\mathbf{A}=\mathbf{C}^{*}$, with $\mathbf{C}$ having weak finite limits, we prove that $\mathbf{A}^{+}$has enough projectives (see Theorem 2.1). The main result of the paper is formulated in Theorem 4.1. The 'finitary' case of it says that, for any small category $\mathbf{C}$ with weak finite limits, the free exact completion of $\mathbf{C}$ is $\mathbf{C}^{*+}$, the category of those set-valued functors of $\mathbf{C}^{*}$ which preserve small products and filtered colimits. In order to prove freeness, we need to extend the notion of flat functor from set-valued functors to functors with arbitrary Barr-exact codomain (see Definition 3.1).

This work extends Makkai's work on 'A theorem on Barr-exact categories' and the author's previous work on 'Dualities for accessible categories', see [11, 8]. The approach is the same as the one taken in Barr [3] and Makkai [11]. A theorem of Barr [3] (Proposition 1.2) is used in the proof of our main result (Theorem 4.1).

Throughout the paper the condition 'finite' is traded for 'less than $\kappa$ ', with an arbitrary regular cardinal number $\kappa$.

\section{A duality theorem for $\kappa$-accessible categories with products}

Let $\kappa$ be an infinite regular cardinal. Recall from [7] that an object $A$ of a category $\mathbf{A}$ is said to be $\kappa$-presentable if the representable functor $\mathbf{A}(A,-)$ preserves $\kappa$-filtered colimits existing in $\mathbf{A}$. $\mathbf{A}$ is $\kappa$-accessible if: (i) $\mathbf{A}$ has $\kappa$-filtered colimits; (ii) there is a small subcategory $\mathbf{C}$ of $\mathbf{A}$ consisting of $\kappa$-presentable objects such that every object of $\mathbf{A}$ is a $\kappa$-filtered colimit of a diagram of objects in $\mathbf{C}$. A category is accessible if it is $\kappa$-accessible for some $\kappa$. A functor $F: \mathbf{A} \rightarrow \mathbf{B}$ is $\kappa$-accessible if $\mathbf{A}$ and $\mathbf{B}$ are $\kappa$-accessible categories and $F$ preserves $\kappa$-filtered colimits. A functor between accessible categories is accessible if it is $\kappa$-accessible for some $\kappa$ (see [12]).

The full subcategory of $\mathbf{A}$ whose objects are $\kappa$-presentable is denoted by $\mathbf{A}_{\kappa}$. Recall that for a small category $\mathbf{D}$, a functor $F: \mathbf{D} \rightarrow$ Set is $\kappa$-flat, if it is a $\kappa$-filtered colimit of representable functors (see $[9,12])$. As shown in [12], a category $\mathbf{A}$ is $\kappa$-accessible if and only if it is equivalent to the category of the form $\kappa$-Flat(D) with $\mathbf{D}$ small; here $\kappa$-Flat $(D)$ is the category of $\kappa$-flat functors from D to Set. 
A $\kappa$-accessible category with products is called weakly locally $\kappa$-presentable in [1]. For a $\kappa$-accessible category $\mathbf{A}$ having products, $\prod$ Filt $_{\kappa}(\mathbf{A}, \mathbf{S e t})$ denotes the category of functors from $A$ to Set that preserve $\kappa$-filtered colimits and products.

Recall from [2] that a category is exact if it has finite limits and stable quotients of equivalence relations. A functor between exact categories is regular if it preserves finite limits and quotients of equivalence relations. The notions of $\kappa$-Barr-exact category and $\kappa$-regular functor are introduced in [11], for $\kappa$ any infinite regular cardinal. They are a natural generalization of the notions of exact category and regular functor.

DefinITION 1.1. ([11]) A category $\mathbf{D}$ is $\kappa$-Barr-exact if it is exact, has $\kappa$-limits, and satisfies the principle of $<\kappa$ dependent choices $\left(D C_{\kappa}\right)$ : let $\alpha$ be an ordinal less than $\kappa$, and let $\Gamma=\left\langle A_{\beta}, f_{\beta, \gamma}: A_{\beta} \rightarrow A_{\gamma}\right\rangle_{\gamma \leq \beta<\alpha}$ be an inverse diagram of type $\alpha$ in $\mathbf{D}$ such that

(i) $f_{\beta+1, \beta}$ is a regular epi, for every $\beta$ with $\beta+1<\alpha$; and

(ii) the restriction $\Gamma \mid \leq \beta$ of $\Gamma$ to the domain consisting of all ordinals $\gamma \leq \beta$ is a limit diagram: $A_{\beta}$ is a limit of $\Gamma \mid<\beta$ ( $\Gamma$ restricted to ordinals $<\beta$ ) with limit projections $f_{\beta, \gamma}: A_{\beta} \rightarrow A_{\gamma}(\gamma<\beta)$, for every limit ordinal $\beta<\alpha$.

Then every $f_{\beta, \gamma}$ is a regular epi, for all $\gamma \leq \beta<\alpha$.

A functor between $\kappa$-Barr-exact categories is $\kappa$-regular, if it preserves all regular epis and all $\kappa$-limits. $\kappa-\operatorname{Reg}(\mathbf{B}, \mathbf{D})$ denotes the category of $\kappa$-regular functors from $\mathbf{B}$ to $\mathbf{D}$.

The following result will be used in Section 4. The result is due to M. Barr for the case $\kappa=\aleph_{0}$ (see [3]).

PROPOSITION 1.2. ([11, Proposition 6.3]) Let $\mathbf{D}$ be a small $\kappa$-Barr-exact category. Then for any functor $M: \mathbf{D} \rightarrow$ Set preserving $\kappa$-limits, there are $N \in \kappa-\operatorname{Reg}(\mathbf{D}$, Set) and a regular monomorphism $M \rightarrow N$ in $L_{\kappa}(\mathbf{D}$, Set $)$, the category of functors from D to Set that preserve $\kappa$-limits. Therefore, $M$ is the domain of an equalizer of a pair of morphisms in $\kappa-\operatorname{Reg}(\mathbf{D}$, Set $)$.

We now Let $\mathbf{C}$ to be a small category with weak $\kappa$-limits.

PROPOSITION 1.3. The category $\kappa$-Flat $(\mathbf{C})$ is $\kappa$-accessible with products.

PROOF. The $\kappa$-accessibility of $\kappa$-Flat(C) is given by [12, Proposition 2.1.4]. To show that $\kappa$-Flat $(\mathbf{C})$ has products, we only need to check that $\kappa$-Flat $(\mathbf{C})$ is closed under products in $(\mathrm{C}$, Set).

Let $\left\langle F_{i}: \mathbf{C} \rightarrow \text { Set }\right\rangle_{i \in l}$ be a small set of $\kappa$-flat functors. Note that a functor $F$ is $\kappa$-flat if and only if the category el $(F)$ of elements of $F$ is $\kappa$-filtered (see $[9,12])$. We will verify that el $(M)$, with $M=\prod_{i \in I} F_{i}$, is $\kappa$-filtered since each $\mathrm{el}\left(F_{i}\right)$ is $\kappa$-filtered. 
Let $K$ be a graph of size less than $\kappa$, and let

$$
G: K \longrightarrow \operatorname{el}(M)
$$

be any diagram with $G(k)=\left(C_{k},\left\langle x_{i, k}\right\rangle_{i \in I}\right)$ and each $x_{i, k}$ in $F_{i}\left(C_{k}\right)$. Since el $\left(F_{i}\right)$ is $\kappa$-filtered there are $D_{i}$ and a cocone

$$
\left\langle f_{i, k}: x_{i, k} \in F_{i}\left(C_{k}\right) \rightarrow y_{i} \in F_{i}\left(D_{i}\right)\right\rangle_{k \in K}
$$

for some $y_{i} \in F_{i}\left(D_{i}\right)$, so that $F_{i}\left(f_{i, k}\right)\left(y_{i}\right)=x_{i, k}$ for each $k \in K$. Since $\mathbf{C}$ has weak $\kappa$-limits, there are $C \in \mathbf{C}$ and a cone $\left\langle g_{k}: C \rightarrow C_{k}\right\rangle_{k \in K}$ in $\mathbf{C}$ such that each $f_{i, k}$ factors through $g_{k}$ for all $k \in K$. Hence we obtain a cocone

$$
\left\langle g_{k}:\left\langle x_{i, k}\right\rangle \in M\left(C_{k}\right) \rightarrow c \in M(C)\right\rangle_{k \in K}
$$

with $c=\left\langle y_{i}\right\rangle_{i \in I}$.

Proposition 1.4. ([8, Proposition 5.5]) Let $\mathbf{A}$ be a $\kappa$-accessible category with small products. Then for any functor $F$ in $\prod$ Filt ${ }_{\kappa}(\mathbf{A}$, Set), there are a $\kappa$-presentable object $A$ in $\mathbf{A}$ and a regular epimorphism $\eta: \mathbf{A}(A,-) \rightarrow F$. Moreover, every $F \in \prod$ Filt ${ }_{\kappa}(\mathbf{A}$, Set) is the codomain of a coequalizer of a pair of morphisms between representable functors.

PROOF. Let $\mathbf{B}$ be a small full subcategory of $\mathbf{A}$ consisting of $\kappa$-presentable objects so that every object of $\mathbf{A}$ is a $\kappa$-filtered colimit of a diagram of objects in $\mathbf{B}$. Given a functor $F \in \prod$ Filt $_{\kappa}$ (A, Set), for every $B \in \mathbf{B}$, let us enumerate all elements of $F(B)$ as $\left\langle a_{i}^{B}\right\rangle_{i \in J_{B}}$ with $J_{B}$ an ordinal number. Consider the small product $\prod_{B \in \mathbf{B}} B^{J_{B}}$ in $\mathbf{A}$. The product is the colimit of a $\kappa$-filtered diagram $\left(\left\langle B_{s}\right\rangle_{s \in S},\left\langle a_{s t}: B_{s} \rightarrow B_{t}\right\rangle_{s \leq t}\right)$ with colimit injections $<e_{s}: B_{s} \rightarrow \prod_{B \in \mathbf{B}} B^{J_{B}}>_{s \in S}$ and with $B_{s}$ in B. Let $K$ be the join of all $J_{B}$, and let $\left\langle a_{k}\right\rangle_{k \in K}$ be the set of elements of the join of all $F(B), B \in \mathbf{B}$. Since $F$ preserves products, then $F\left(\prod_{B \in \mathbf{B}} B^{J_{B}}\right) \cong \prod_{B \in \mathbf{B}} F(B)^{J_{B}}$, and $F$ maps the product projections $\pi_{k}: \prod_{B \in \mathbf{B}} B^{J_{B}} \rightarrow B$ to the projections in Set. Hence there is $a \in F\left(\prod_{B \in \mathbf{B}} B^{J_{B}}\right)$ such that $F\left(\pi_{k}\right)(a)=a_{k}$, for all $k \in K$. Also note that since $F$ preserves $\kappa$-filtered colimits, the morphisms $F\left(e_{s}\right): F\left(B_{s}\right) \rightarrow F\left(\prod_{B \in \mathbf{B}} B^{J_{B}}\right)$ make $F\left(\prod_{B \in \mathbf{B}} B^{J_{B}}\right)$ a $\kappa$-filtered colimit of the diagram $\left(\left\langle F\left(B_{s}\right)\right\rangle_{s \in S},\left\langle F\left(a_{s t}\right)\right\rangle_{s \leq t}\right)$ in Set. Thus there is $s \in S$ and some $c \in F\left(B_{s}\right)$ such that $F\left(e_{s}\right)(c)=a$. It follows that

$$
F\left(\pi_{k} \circ e_{s}\right)(c)=a_{k}, \text { for every } k \in K .
$$

We use $A$ for $B_{s}$. The Yoneda lemma gives a natural transformation $\eta: \mathbf{A}(A,-) \longrightarrow$ $F$ with $\eta_{A}\left(\mathrm{id}_{A}\right)=c$. For every $B \in \mathbf{B}$, we have that $\eta_{B}$ is surjective in Set. Since every object of $\mathbf{A}$ is a $\kappa$-filtered colimit of objects in $\mathbf{B}$, and $A$ is $\kappa$-presentable, it 
is easy to see that $\eta_{A^{\prime}}$ is surjective for all $A^{\prime} \in \mathbf{A}$. $\prod$ Filt ${ }_{\kappa}(\mathbf{A}$, Set) is exact and the inclusion $\prod$ Filt $_{\kappa}(\mathbf{A}$, Set $) \rightarrow(\mathbf{A}$, Set) is regular; in the latter category, $\eta$ is a regular epimorphism means that $\eta_{A^{\prime}}$ is surjective for all $A^{\prime} \in \mathbf{A}$. We conclude that $\eta$ is a regular epimorphism in $\prod$ Filt $_{\kappa}(\mathbf{A}$, Set).

We have established that every $F \in \prod$ Filt $_{\kappa}$ (A, Set) admits a regular epimorphism from a representable functor $\mathbf{A}(A,-)$ to $F$, with $A \in \mathbf{B}$. Thus we have a coequalizer diagram

$$
G \underset{g}{\stackrel{f}{\longrightarrow}} \mathbf{A}(A,-) \stackrel{\eta}{\longrightarrow} F
$$

Using the previous argument again, we obtain a regular epimorphism $e: \mathbf{A}(B,-)$ $\rightarrow G$ with $B \in \mathbf{B}$, and $\eta$ a coequalizer of the morphisms $(f \circ e, g \circ e$ ) between the representable functors $\mathbf{A}(A,-)$ and $\mathbf{A}(B,-)$.

Corollary 1.5. For A in Proposition 1.4, $\prod$ Filt $_{\kappa}(\mathbf{A}$, Set) is small $\kappa$-Barr-exact.

Proof. The smallness of $\prod$ Filt ${ }_{\kappa}$ (A, Set) follows from the smallness of $\mathbf{A}_{\kappa}$, by Proposition 1.4.

COROLlary 1.6. For any $\kappa$-accessible category $\mathbf{A}$ with products, $\mathbf{A}_{\kappa}^{\text {op }}$ has weak $\kappa$-limits.

PROOF. Let $G: I \rightarrow \mathbf{A}_{\kappa}^{o p}$ be a $\kappa$-diagram, and let $y: \mathbf{A}_{\kappa}^{o p} \rightarrow \prod$ Filt $_{\kappa}(\mathbf{A}$, Set) be the restricted Yoneda embedding. Since $\prod$ Filt ${ }_{\kappa}(\mathbf{A}$, Set $)$ has $\kappa$-limits, we let $\left\langle p_{i}: M \rightarrow \mathbf{A}\left(A_{i},-\right)\right\rangle_{i \in I}$ be the projections of $\lim (y \circ G)$. From Proposition 1.4, we obtain some $A \in \mathbf{A}_{\kappa}$ and a regular epi $e: \mathbf{A}(A,-) \rightarrow M$. For any cone $\left\langle q_{i}: \mathbf{A}(B,-) \rightarrow \mathbf{A}\left(A_{i},-\right)\right\rangle_{i \in l}$ with $B \in \mathbf{A}_{\kappa}$, by the projectivity of $\mathbf{A}(B,-)$, there is a morphism $t: \mathbf{A}(B,-) \rightarrow \mathbf{A}(A,-)$ such that $q_{i}=\left(p_{i} \circ e\right) \circ t$ for all $q_{i}$. We conclude that $\mathbf{A}_{\kappa}^{o p}$ has weak $\kappa$-limits from the Yoneda lemma.

The following duality theorem for $\kappa$-accessible categories with products is now obtained easily.

THEOREM 1.7. A category is $\kappa$-accessible and has products if and only if it is equivalent to the category of the form $\kappa$-Flat $(\mathbf{C})$, for some small category $\mathbf{C}$ with weak $\kappa$-limits.

PROOF. For a $\kappa$-accessible category $\mathbf{A}$, one already has the equivalence $\mathbf{A} \simeq$ $\kappa$-Flat $\left(\mathbf{A}_{\kappa}^{o p}\right)$ (see [12]). Thus, Theorem 1.7 follows from From Proposition 1.3 and Corollary 1.6. 
COROLlaRY 1.8. ([1, Theorem I.6]) An accessible category $\mathbf{A}$ has products if and only if it has weak colimits.

PROOF. Let $\mathbf{A}$ be $\boldsymbol{\kappa}$-accessible. If $\mathbf{A}$ has weak colimits, then $\mathbf{A}_{\kappa}$ has weak $\boldsymbol{\kappa}$-colimits, thus $\mathbf{A}$ has products. Conversely, if $\mathbf{A}$ has products, for any regular cardinal $\lambda>\kappa$, we have a regular cardinal $\lambda^{\prime}>\lambda$ such that $\mathbf{A}$ is $\lambda^{\prime}$-accessible (see [12, Theorem 2.3.10]). Thus, $\mathbf{A}_{\lambda^{\prime}}$ has weak $\lambda^{\prime}$-colimits. Consequently, $\mathbf{A}$ has weak colimits.

\section{Canonical $\kappa$-flat functors on a small category with weak $\kappa$-limits}

For any small category $\mathrm{C}$ with weak $\kappa$-limits, by Proposition $1.3, \kappa$-Flat $(\mathrm{C})$ is $\kappa$-accessible with products. We denoted $\kappa$-Flat $(\mathbf{C})$ by $\mathbf{C}^{*}$. Let $\prod$ Filt ${ }_{\kappa}\left(\mathbf{C}^{*}\right.$, Set $)$ be the category of all functors from $\mathrm{C}^{*}$ to Set that preserve $\kappa$-filtered colimits and products; it is denoted by $\mathbf{C}^{*+}$.

We consider the evaluation functor

$$
\mathrm{ev}_{\mathrm{C}}: \mathbf{C} \longrightarrow \text { Filt }_{\kappa}\left(\mathbf{C}^{*}, \text { Set }\right) .
$$

Since $\mathbf{C}^{*}$ is the free $\kappa$-filtered colimit completion of $\mathbf{C}^{o p}$ (see $\left.[9,12]\right)$, the functor

$$
I: \text { Filt }_{\kappa}\left(\mathrm{C}^{*}, \text { Set }\right) \rightarrow\left(\mathbf{C}^{o p}, \text { Set }\right)
$$

induced by the opposite Yoneda embedding $Y^{\prime}: \mathbf{C}^{o p} \rightarrow \kappa$-Flat(C) is an equivalence. Let $I^{\prime}$ be the quasi-inverse of $I$, then $\operatorname{ev}_{\mathbf{C}}=I^{\prime} \circ Y$; here $Y: \mathbf{C} \rightarrow\left(\mathbf{C}^{o p}\right.$, Set $)$ is the Yoneda embedding. Hence $\mathrm{ev}_{\mathrm{C}}$ is full and faithful. For every $C \in \mathbf{C}$, since $\mathrm{ev}_{\mathrm{C}}(C)$ preserves products, so $\mathrm{ev}_{\mathrm{C}}$ induces a functor

$$
e_{\mathbf{C}}: \mathbf{C} \rightarrow \mathbf{C}^{*+} .
$$

For every $C \in \mathrm{C}$, note that $e_{\mathrm{C}}(C)$ is projective in $\mathrm{C}^{*+}$. From Proposition 1.4, we have

THEOREM 2.1. For every small category $\mathbf{C}$ with weak $\kappa$-limits, the evaluation functor $e_{\mathrm{C}}$ has the following properties:

(i) $e_{\mathrm{C}}$ is full and faithful;

(ii) $e_{\mathbf{C}}(C)$ is projective in $\mathbf{C}^{*+}$, for any $C \in \mathbf{C}$;

(iii) for each $F \in \mathbf{C}^{*+}$, there are $C \in \mathbf{C}$ and a regular epimorphism $\eta: e_{\mathbf{C}}(C) \rightarrow$ $F$ in $\mathbf{C}^{*+}$.

COROLLARY 2.2. The functor $e_{\mathrm{C}}$ is dense.

ProOF. From [3, Theorem 14] or [8, Proposition 5.3]. 
PROPOSITION 2.3. The functor

$$
\begin{gathered}
Z: \kappa-\operatorname{Reg}\left(\mathbf{C}^{*+}, \text { Set }\right) \longrightarrow(\mathbf{C}, \text { Set }) \\
M \longmapsto M \circ e_{\mathbf{C}}
\end{gathered}
$$

is full and faithful, and its essential image consists of the $\kappa$-flat functors from $\mathbf{C}$ to Set, that is, we have an equivalence

$$
\kappa-\operatorname{Reg}\left(\mathbf{C}^{*+}, \text { Set }\right) \simeq \kappa-\operatorname{Flat}(\mathbf{C}) .
$$

PROOF. From [8, Proposition 5.8] and Theorem 2.1.

\section{3. $\kappa$-flat functors}

In this section, $\mathbf{B}$ denotes a $\kappa$-Barr-exact category. We identify $\mathbf{C}$ with its image under $e_{\mathrm{C}}$ in Theorem 2.1. The inclusion functor $\mathbf{C} \rightarrow \mathbf{C}^{*+}$ is denoted by $l$, so the equivalent conditions of Theorem 2.1 are satisfied by $l$.

DEFINITION 3.1. Let $\mathbf{C}$ be an arbitrary category, and $\mathbf{B}$ a $\kappa$-Barr-exact category. A functor $F: \mathbf{C} \rightarrow \mathbf{B}$ is called $\kappa$-flat if, for any $\kappa$-diagram $G: I \rightarrow \mathbf{C}$, there is a cone $\left(f_{i}: D \rightarrow G(i)\right)_{i \in l}$ on $G$ such that $F\left(f_{i}\right)=p_{i} \circ k$ for all $i \in I$ and the morphism $k: F(D) \rightarrow \lim F \circ G$ is a regular epi; here the morphisms $p_{i}$ are limits projections.

Remark 3.2. (i) In the case that $\mathbf{C}$ is small and $\mathbf{B}$ is the category Set, it is easy to check that Definition 3.1 is equivalent to the original concept whereby $F$ is $\kappa$-flat if and only if it is a $\kappa$-filtered colimit of representable functors.

(ii) In the case that $\mathbf{C}$ is small with $\kappa$-limits, a functor between $\mathbf{C}$ and $\mathbf{B}$ preserves $\kappa$-limits if and only if it is $\kappa$-flat. The necessary condition of this statement is trivial, the proof of the sufficient condition will be given by Theorem 4.1 (see Corollary 4.3).

We now assume that $\mathbf{C}$ is a small category with weak $\kappa$-limits. We use $\kappa$-Flat $(\mathbf{C}, \mathbf{B})$ to denote the full subcategory of $(\mathbf{C}, \mathbf{B})$ whose objects are $\kappa$-flat functors. We have

PROPOSITION 3.3. e ef Theorem 2.1 is $\kappa$-flat.

PROOF. From Theorem 2.1.

Proposition 3.4. For any $\kappa-B$ arr-exact category $\mathbf{B}$, if $M \in \kappa-\operatorname{Reg}\left(\mathbf{C}^{*+}, \mathbf{B}\right)$ then $M \circ e_{\mathbf{C}} \in \kappa-$ Flat $(\mathbf{C}, \mathbf{B})$.

PROOF. From Definition 3.1 and Proposition 3.3. 
THEOREM 3.5. Every $\kappa$-flat functor $F: \mathbf{C} \rightarrow \mathbf{B}$ has a left Kan extension $F$ ! along $l$ and $F$ ! preserves regular epimorphisms; here $l$ is the inclusion mentioned in the beginning of the section.

PROOF. For the existence of $F$ !, by the dual of [10, Theorem X.3.1], it suffices to show that the composite $F \circ P: l / C^{\prime} \rightarrow \mathbf{C} \rightarrow \mathbf{B}$ has a colimit in $\mathbf{B}$ for each $C^{\prime} \in \mathbf{C}^{*+}$, where $P$ is the projection $\left\langle C, C \rightarrow C^{\prime}\right\rangle \mapsto C$. Since $C^{\prime} \in \mathrm{C}^{*+}$, according to Theorem 2.1, we can take a regular epimorphism $e: A \rightarrow C^{\prime}$ with $A$ in $\mathrm{C}$, and let

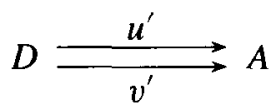

be the kernel pair of $e$; so $e$ is the coequalizer of $\left(u^{\prime}, v^{\prime}\right)$. Let $d: S \rightarrow D$ be a regular epimorphism, then $e$ is a coequalizer of the morphisms $\left(u^{\prime} \circ d, v^{\prime} \circ d\right)$. Denote $u^{\prime} \circ d$ by $u$, and $v^{\prime} \circ d$ by $v$. Consider the category $\mathbf{E}$ as follows.

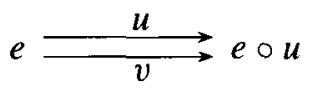

Let $i: E \rightarrow l / C^{\prime}$ be the inclusion. We have

LEMMA 3.6. $i$ is final.

PROOF. Firstly, $f / i$ is non-empty, for any $f: C \rightarrow C^{\prime}$ with $C \in \mathbf{C}$. Indeed, by the projectivity of $C$, there is a morphism $w: C \rightarrow A$ such that $f=e \circ w$.

To show that $f / i$ is connected, let $m, n$ be any two morphisms in $f / i$. Then we only need to consider the following three cases.

Case 1: $m, n: f \rightarrow e$, that is, $e \circ m=e \circ n=f$. Since $\left\langle u^{\prime}, v^{\prime}\right\rangle$ is the kernel pair of $e$, there is a unique morphism $k^{\prime}: C \rightarrow D$ such that $m=u^{\prime} \circ k^{\prime}$ and $n=v^{\prime} \circ k^{\prime}$. By the projectivity of $C$ we obtain a morphism $k: C \rightarrow S$ with $k^{\prime}=d \circ k$. Thus $m=u \circ k$ and $n=v \circ k$, that is, $u: k \rightarrow m$ and $v: k \rightarrow n$; here $k: f \rightarrow e \circ u$ is in $f / i$.

Case 2: $m, n: f \rightarrow e \circ u$, that is, $e \circ u^{\prime} \circ d \circ m=e \circ v^{\prime} \circ d \circ n=f$. Since $\left\langle u^{\prime}, v^{\prime}\right\rangle$ is the kernel pair of $e$, there is a unique morphism $k^{\prime}: C \rightarrow D$ such that $u^{\prime} \circ d \circ m=u^{\prime} \circ k^{\prime}$ and $v^{\prime} \circ d \circ n=v^{\prime} \circ k^{\prime}$. By the projectivity of $C$, we have a morphism $k: C \rightarrow S$ with $k=d \circ k^{\prime}$. We conclude that $u \circ m=u \circ k$ and $v \circ n=v \circ k$. Thus, we have four morphisms $u: m \rightarrow u \circ k, u: k \rightarrow u \circ k, v: n \rightarrow v \circ k$ and $v: k \rightarrow v \circ k$, joining $m$ to $n$.

Case 3: $m: f \rightarrow e$ and $n: f \rightarrow e \circ u$. By the projectivity of $C$, there is a morphism $m^{\prime}: f \rightarrow e \circ u$ such that $m=u \circ m^{\prime}$, because $u$ is regular epi. Thus, we have morphisms $m^{\prime}, n: f \rightarrow e \circ u$. That $f / i$ is connected now follows from the Case 2. This completes the proof of that $f / i$ is connected. 
We continue the proof of Theorem 3.5. Since $i$ is final, according to [10, Theorem IX.3.1], to prove that $F$ ! exists, we only need to show that the pair of morphisms $(F(u), F(v))$ has a coequalizer in $\mathbf{B}$.

Let $(p, q)$ be the product projections of $F(A) \prod F(A)$, and let $\epsilon: F(S) \rightarrow$ $F(A) \prod F(A)$ be the unique morphism so that $F(u)=p \circ \epsilon$ and $F(v)=q \circ \epsilon$. Since B is $\kappa$-Barr-exact, $\epsilon$ has a factorization $\epsilon=y \circ x$ with $y: Q \rightarrow F(A) \prod F(A)$ mono and $x: F(S) \rightarrow Q$ regular epi, for some $Q \in \mathbf{B}$.

LEMMA 3.7. $y$ is an equivalence relation on $F(A)$.

ProOF. (i) (Reflexivity) The diagonal $\Delta: F(A) \rightarrow F(A) \prod F(A)$ factors through $y$.

Let $k^{\prime}: A \rightarrow D$ be the morphism so that $i d_{A}=u^{\prime} \circ k^{\prime}=v^{\prime} \circ k^{\prime}$. Since $S$ is projective, one obtain a morphism $k: A \rightarrow S$ with $k^{\prime}=d \circ k$, hence we have that $i d_{A}=u \circ k=v \circ k$. It follows that

$$
i d_{F(A)}=F(u) \circ F(k)=p \circ y \circ x \circ F(k)=q \circ y \circ x \circ F(k) .
$$

Consequently, $\Delta=y \circ(x \circ F(k))$.

(ii) (Symmetry) There exists a morphism $t: Q \rightarrow Q$ such that $p \circ y=q \circ y \circ t$ and $q \circ y=p \circ y \circ t$.

Let $\left(\pi_{1}, \pi_{2}\right)$ be the product projections of $A \prod A$, and let $m: D \rightarrow A \prod A$ be the induced morphism of $\left(u^{\prime}, v^{\prime}\right)$. Since the kernel pair of a morphism always yields an equivalence relation, there exists $n: D \rightarrow D$ such that $\pi_{1} \circ m=\pi_{2} \circ m \circ n$ and $\pi_{2} \circ m=\pi_{1} \circ m \circ n$. Since $d: S \rightarrow D$ is regular epi, by the projectivity of $S$, there is a morphism $n^{\prime}: S \rightarrow S$ such that $n \circ d=d \circ n^{\prime}$. Thus, we have that $\pi_{1} \circ m \circ d \circ n^{\prime}=\pi_{2} \circ m \circ d$ and $\pi_{2} \circ m \circ d \circ n^{\prime}=\pi_{1} \circ m \circ d$, that is, $u \circ n^{\prime}=v$ and $v \circ n^{\prime}=u$. Applying $F$ to the above equalities, then $F(u) \circ F\left(n^{\prime}\right)=F(v)$ and $F(v) \circ F\left(n^{\prime}\right)=F(u)$. We obtain that

$$
p \circ y \circ x \circ F\left(n^{\prime}\right)=q \circ y \circ x \text { and } q \circ y \circ x \circ F\left(n^{\prime}\right)=p \circ y \circ x .
$$

Let $(f, g)$ be the kernel pair of $x$. Then,

$$
p \circ y \circ x \circ F\left(n^{\prime}\right) \circ f=q \circ y \circ x \circ f=q \circ y \circ x \circ g=p \circ y \circ x \circ F\left(n^{\prime}\right) \circ g .
$$

Similarly,

$$
q \circ y \circ x \circ F\left(n^{\prime}\right) \circ f=q \circ y \circ x \circ F\left(n^{\prime}\right) \circ g .
$$

It follows that $y \circ x \circ F\left(n^{\prime}\right) \circ f=y \circ x \circ F\left(n^{\prime}\right) \circ g$ from the product projections $(p, q)$. But $y$ is mono, so $x \circ F\left(n^{\prime}\right) \circ f=x \circ F\left(n^{\prime}\right) \circ g$. Since $(f, g)$ is the coequalizer of $x$, there is a unique morphism $t: Q \rightarrow Q$ such that $t \circ x=x \circ F\left(n^{\prime}\right)$. It is easily seen that $t$ is as required. 
(iii) (Transitivity) For the pullback diagram of $p \circ y$ and $q \circ y$

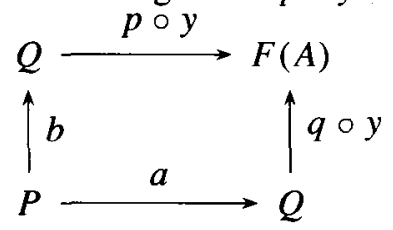

the morphism $\delta=\langle(p \circ y) \circ a,(q \circ y) \circ b\rangle: P \rightarrow F(A) \prod F(A)$ factors through $y$.

Let $(z, w: U \rightarrow F(S))$ be the pullback of $F(u)$ and $F(v)$. There is a unique morphism $\alpha: U \rightarrow P$ such that $x \circ z=b \circ \alpha$ and $x \circ w=a \circ \alpha$. Then $\alpha$ is a regular epi. In fact, let $b^{\prime}: U_{1} \rightarrow F(S)$ and $x_{1}: U_{1} \rightarrow P$ be the pullback of $x$ and $b$ and let $x_{2}: U_{2} \rightarrow P$ and $a^{\prime}: U_{2} \rightarrow F(S)$ be the pullback of $a$ and $x$. Since $x$ is regular epi so are $x_{1}$ and $x_{2}$. Let $x_{2}^{\prime}$ and $x_{1}^{\prime}$ be the pullback of $x_{1}$ and $x_{2}$, then $b^{\prime} \circ x_{2}^{\prime}$ and $a^{\prime} \circ x_{1}^{\prime}$ is the pullback of $F(u)$ and $F(v)$. Therefore $\alpha=x_{1} \circ x_{2}^{\prime}=x_{2} \circ x_{1}^{\prime}$. That $\alpha$ is regular epi follows from the fact that $x_{1}$ and $x_{2}^{\prime}$ are regular epi.

Since $F$ is $\kappa$-flat, from Definition 3.1, there are two morphisms $s, t: V \rightarrow S$ in C with $u \circ s=v \circ t$ such that $F(s)=z \circ \beta$ and $f(t)=w \circ \beta$ for some regular epi $\beta: F(V) \rightarrow U$ in $\mathbf{B}$. Thus, $\alpha \circ \beta: F(V) \rightarrow P$ is a regular epi in $\mathbf{B}$.

Note that $\left(u^{\prime}, v^{\prime}\right)$ is an equivalence relation on $A$, let

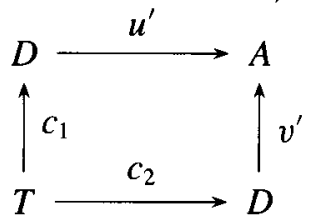

be the pullback diagram of $u^{\prime}$ and $v^{\prime}$. Thus, the morphism $c=\left\langle u^{\prime} \circ c_{2}, v^{\prime} \circ c_{1}\right\rangle: T \rightarrow$ $A \prod A$ factors as $c=m \circ r^{\prime}$ for some $r^{\prime}: T \rightarrow D$; here $m$ is given in the proof of the symmetry. Since $u \circ s=v \circ t$, that is, $u^{\prime} \circ d \circ s=v^{\prime} \circ d \circ t$, there exists a unique morphism $d^{\prime}: V \rightarrow T$ such that $d \circ s=c_{1} \circ d^{\prime}$ and $d \circ t=c_{2} \circ d^{\prime}$. By the projectivity of $V$, we have $r: V \rightarrow S$ with $d \circ r=r^{\prime} \circ d^{\prime}$. Since

$$
\begin{aligned}
u^{\prime} \circ d \circ t & =u^{\prime} \circ c_{2} \circ d^{\prime}=\pi_{1} \circ c \circ d^{\prime} \\
& =\pi_{1} \circ m \circ r^{\prime} \circ d^{\prime}=u^{\prime} \circ d \circ r,
\end{aligned}
$$

then $u \circ t=u \circ r$. Similarly $v \circ s=v \circ r$. Apply $F$ to the above equalities, then $F(u) \circ F(t)=F(u) \circ F(r)$ and $F(v) \circ F(s)=F(v) \circ F(r)$. Since $F(u)=p \circ y \circ x$ and $F(v)=q \circ y \circ x$, it follows that

$$
p \circ y \circ x \circ F(r)=p \circ y \circ x \circ F(t)=p \circ y \circ x \circ w \circ \beta=p \circ y \circ a \circ \alpha \circ \beta
$$

and $q \circ y \circ x \circ F(r)=q \circ y \circ b \circ \alpha \circ \beta$. Let $\left(f^{\prime}, g^{\prime}\right)$ be the kernel pair of $\alpha \circ \beta$. Then,

$$
\begin{aligned}
& p \circ y \circ x \circ F(r) \circ f^{\prime}=p \circ y \circ x \circ F(r) \circ g^{\prime}, \\
& q \circ y \circ x \circ F(r) \circ f^{\prime}=q \circ y \circ x \circ F(r) \circ g^{\prime} .
\end{aligned}
$$


It follows that $y \circ x \circ F(r) \circ f^{\prime}=y \circ x \circ F(r) \circ g^{\prime}$ as $p$ and $q$ are projections and $x \circ F(r) \circ f^{\prime}=x \circ F(r) \circ g^{\prime}$ by the fact that $y$ is mono. Since $\alpha \circ \beta$ is the coequalizer of $f^{\prime}$ and $g^{\prime}$ there is a unique morphism $\eta: P \rightarrow Q$ such that $\eta \circ \alpha \circ \beta=x \circ F(r)$. Thus $p \circ y \circ a=p \circ y \circ \eta$ and $q \circ y \circ b=q \circ y \circ \eta$. Consequently $\delta=y \circ \eta$.

We now turn to the proof of Theorem 3.5. Since B is $\kappa$-Barr-exact, every equivalence relation is effective, so $(p \circ y, q \circ y)$ has a coequalizer. Since $F(u)=p \circ y \circ x$ and $F(v)=q \circ y \circ x$, it follows that $(F(u), F(v))$ has a coequalizer as $x$ is regular epi. This completes the proof of the existence of $F$ !.

From the above proof, we see that $F$ ! takes any regular epi with domain in $\mathbf{C}$ into regular epi. Indeed, given a regular epi $e: P \rightarrow Q$ in $\mathbf{C}^{*+}$, we take a regular epi $d: C \rightarrow P$ with $C \in \mathbf{C}$. Since $F(e) \circ F(d)$ is a regular epi, so is $F(e)$.

REMARK 3.8. From the proof of Theorem 3.5, we see that the left Kan extension $F$ ! of Theorem 3.5 preserves the coequalizer of the kernel pair of any regular epi. We will study such functors in a forthcoming paper.

\section{Exact completion of weak-lex categories}

THEOREM 4.1. Let $\mathbf{C}$ be a small category with weak $\kappa$-limits. Then the canonical functor $e_{\mathbf{C}}: \mathbf{C} \rightarrow \mathbf{C}^{*+}$ has the following universal property which characterizes it as the free $\kappa$-Barr-exact completion of $\mathbf{C}$.

(i) For any $\kappa$-Barr-exact category $\mathbf{B}$, the functor

$$
\begin{gathered}
\Sigma: \kappa-\operatorname{Reg}\left(\mathbf{C}^{*+}, \mathbf{B}\right) \rightarrow \kappa-\operatorname{Flat}(\mathbf{C}, \mathbf{B}) \\
M \mapsto M \circ e_{\mathbf{C}}
\end{gathered}
$$

induced by $e_{\mathrm{C}}$ is an equivalence of categories.

(ii) The quasi-inverse of the equivalence $\Sigma$ of (i) takes a $\kappa$-flat functor $F: \mathbf{C} \rightarrow \mathbf{B}$ to its left Kan extension $F$ ! along $e_{\mathrm{C}}$.

PROOF. The fullness and faithfulness of $\Sigma$ follows from the properties described in Theorem 2.1; for details, see the proof of [8, Proposition 5.8]. We now prove that $\Sigma$ is surjective on objects. Since $\Sigma$ is full and faithful, by [10, Corollary X.3.3], it suffices to show that for any $\kappa$-flat functor $F: \mathbf{C} \rightarrow \mathbf{B}, F$ has a left Kan extension $F$ ! of $F$ along $e_{\mathbf{C}}$, and $F$ ! is $\kappa$-regular. The existence of $F$ ! was shown in Theorem 3.5. Since $F$ ! preserves regular epimorphisms, it remains to show that $F$ ! preserves $\kappa$-limits.

Without loss of generality, we may assume that $\mathbf{B}$ is small, since both of $\mathbf{C}$ and $\mathrm{C}^{*+}$ are essentially small. For any $\kappa$-regular functor $M: \mathbf{B} \rightarrow$ Set, the composite 
$M \circ F: C \rightarrow$ Set is $\kappa$-flat; by Proposition 2.3, we have a unique factorization

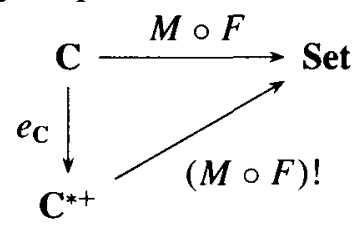

with $(M \circ F)$ ! $\kappa$-regular. Also, note that $F=F ! \circ e_{\mathrm{C}}$. By the uniqueness of the factorization, we conclude that $M \circ F$ ! is $\kappa$-regular.

For any $B \in \mathbf{B}$, we will show that $\mathbf{B}(B,-) \circ F$ ! preserves $\kappa$-limits. Firstly, for any $\kappa$-regular functor $M$, the composite $M \circ F$ ! preserves $\kappa$-limits (it is $\kappa$-regular!). Secondly, every $\mathbf{B}(B,-)$ is the domain of an equalizer of a pair between $\kappa$-regular functors from $\mathbf{B}$ into Set from Proposition 1.2, and from the fact that limits are computed pointwise in $L_{\kappa}(\mathbf{B}$, Set $)$, we conclude that $\mathbf{B}(B,-) \circ F$ ! preserves $\kappa$-limits. Therefore, $F$ ! preserves $\kappa$-limits.

REMARK 4.2. In order to prove Theorem 4.1, we only use the properties of $e_{\mathrm{C}}$ of Theorem 2.1. Therefore, for a $\kappa$-Barr-exact category $\mathbf{D}$, we have that, if there is a functor $F: \mathbf{C} \rightarrow \mathbf{D}$ such that the properties (i), (ii) and (iii) of Theorem 2.1 are satisfied by $F$, then $\mathbf{D}$ is a $\kappa$-Barr-exact completion of $\mathbf{C}$. Consequently, these are necessary and sufficient conditions describing the free $\kappa$-Barr-exact completion of $\mathbf{C}$.

COROLLARY 4.3. Let $\mathbf{C}$ be a small category with $\kappa$-limits. A functor $F: \mathbf{C} \rightarrow \mathbf{B}$ is $\kappa$-flat if and only if it preserves $\kappa$-limits.

Proof. If $F$ is $\kappa$-flat, from Theorem 4.1, $F=F$ ! $\circ e_{\mathrm{C}}$. Since both of $e_{\mathrm{C}}$ and $F$ ! preserve $\kappa$-limits, so does $F$.

The following result is an immediate consequence of Theorem 4.1. For $\kappa=\aleph_{0}$, this result was proved in [4].

COROLlARY 4.4. Let $\mathbf{C}$ be a small category with $\kappa$-limits. Then the canonical functor $e_{C}: \mathbf{C} \rightarrow \prod$ Filt $_{\kappa}\left(L_{\kappa}(\mathbf{C}\right.$, Set $)$, Set $)$ preserves $\kappa$-limits, and $e_{\mathbf{C}}$ has the universal property of a free $\kappa$-Barr-exact completion: for any $\kappa$-Barr-exact category $\mathbf{B}$, every functor $F: \mathbf{C} \rightarrow \mathbf{B}$ preserving $\kappa$-limits can be uniquely (up to isomorphism) factorized as

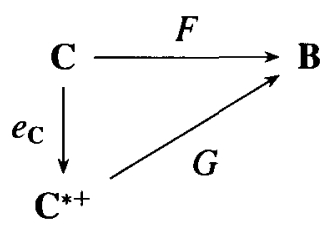

with a $\kappa$-regular $G$. 
Let $\mathbf{C}^{* *}=\prod \operatorname{Acc}\left(\mathbf{C}^{*}\right.$, Set $)$ be the category of accessible functors from $\mathbf{C}^{*}$ to Set that preserve products. The evaluation functor $\epsilon_{\mathbf{C}}: \mathbf{C} \rightarrow \mathbf{C}^{* *}$ is clearly $\kappa$-flat. By [8, Proposition 6.5], $\mathrm{C}^{* *}$ is complete and exact. We have

PROPOSITION 4.5. Let $\mathbf{C}$ be a small category with weak $\kappa$-limits. Then the functor

$$
T: \operatorname{LR}\left(\mathbf{C}^{* \#}, \text { Set }\right) \rightarrow \kappa-F l a t(C, \text { Set })
$$

induced by $\epsilon_{\mathrm{C}}$ is an equivalence; here $\operatorname{LR}\left(\mathbf{C}^{* \#}\right.$, Set $)$ is the category of regular functors from $\mathrm{C}^{* \#}$ to Set that preserve small limits.

Proof. Since $\mathrm{C}^{*}$ is $\kappa$-accessible with products, according to [8, Theorem 6.1(iii)], the evaluation functor $\eta_{\mathbf{C}^{*}}: \mathbf{C}^{*} \rightarrow \operatorname{LR}\left(\mathbf{C}^{* *}\right.$, Set) is an equivalence. But $\operatorname{id}_{\mathbf{C}^{*}} \cong T \circ \eta_{\mathbf{C}^{*}}$, hence $T$ is an equivalence.

THEOREM 4.6. The evaluation functor

$$
\epsilon_{\mathrm{C}}: \mathbf{C} \rightarrow \mathbf{C}^{* \#}
$$

has the following universal property: $\mathbf{C}^{* \#}$ is complete and exact, and for any exact $\mathbf{D}$ with small limits, the functor

$$
\begin{gathered}
\Sigma_{\mathbf{C}}: \operatorname{LR}\left(\mathbf{C}^{* \#}, \mathbf{D}\right) \rightarrow \kappa-\operatorname{Flat}(\mathbf{C}, \mathbf{D}) \\
M \mapsto M \circ \epsilon_{\mathbf{C}}
\end{gathered}
$$

induced by $\epsilon_{\mathrm{C}}$ is an equivalence of categories; here $\operatorname{LR}\left(\mathbf{C}^{* \#}, \mathrm{D}\right)$ is the category of regular functors from $\mathbf{C}^{* \#}$ to $\mathbf{D}$ that preserve small limits.

PROOF. The proof of the theorem is analogous to that of Theorem 4.1.

\section{Acknowledgments}

The work began while the author was visiting Dalhousie University in February 1993. The author is indebted to M. Makkai, W. Tholen and especially R. Paré for stimulating discussions concerning various aspects of this work. The author would also like to thank the referee for his valuable suggestions.

\section{References}

[1] J. Adámek and J. Rosický, 'On weakly locally presentable categories', Cahiers Topologie Géom. Diff. Catégoriques, 35 (1994), 197-186. 
[2] M. Barr, 'Exact categories', in: Lecture Notes in Math. 236 (Springer, Berlin, 1971) pp. 1-120.

[3] — - 'Representations of categories', J. Pure Appl. Algebra 41 (1986), 113-137.

[4] A. Carboni and R. C. Magno, 'The free exact category on a left exact one', J. Austral. Math. Soc. (Ser. A) 33 (1982), 295-301.

[5] A. Carboni, 'Some free constructions in realizability and proof theory', preprint.

[6] P. Freyd, 'Representations in abelian categories', in: Proceedings of the conference on categorical algebra, La Jolla, 1965 (Springer, Berlin, 1966) pp. 95-120.

[7] P. Gabriel and F. Ulmer, Lokal präsentierbare Kategorien, Lecture Notes in Math. 221 (Springer, Berlin, 1971).

[8] H. Hu, 'Dualities for accessible categories', in: Can. Math. Soc. Conference Proceedings 13 (Amer. Math. Soc., Providence, 1992) pp. 211-242.

[9] G. M. Kelly, Basic concepts of enriched category theory (Cambridge University Press, 1982).

[10] S. Mac Lane, Categories for the working mathematician (Springer, Berlin, 1971).

[11] M. Makkai, 'A theorem on Barr-exact categories, with an infinitary generalization', Ann. Pure Appl. Logic 47 (1990), 225-268.

[12] M. Makkai and R. Pare, Accessible categories: the foundations of categorical model theory, Contemporary Math. 104 (Amer. Math. Soc., Providence, 1990).

[13] R. Street, 'Fibrations in bicategories', Cahiers Topologie Géom. Diff. Catégoriques 21 (1980), 111-160.

Département de Mathématiques

Université du Québec à Montréal

Montréal, QC

Canada H3C 3P8

e-mail: hu@math.uqam.ca 